\title{
Correction to: Peripheral and Central Effects of Memantine in a Mixed Preclinical Mice Model of Obesity and Familial Alzheimer's Disease
}

\author{
Miren Ettcheto ${ }^{1,2,3,4}$ • Elena Sánchez-López 2,5,6 Yaiza Gómez-Mínguez ${ }^{1}$. Henrry Cabrera ${ }^{1}$ • Oriol Busquets ${ }^{1,2,3,4}$.

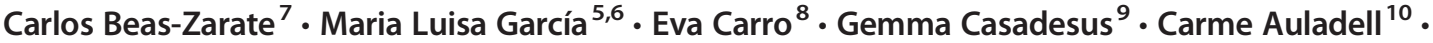 \\ Manuel Vázquez Carrera 1,11,12,13 . Jaume Folch ${ }^{2,3}$. Antoni Camins ${ }^{1,2,4}$ (ID
}

Published online: 4 May 2020

(C) Springer Science+Business Media, LLC, part of Springer Nature 2020

Correction to: Mol Neurobiol (2018) 55, 7327-7339 https://doi.org/10.1007/s12035-018-0868-4

The original version of this article unfortunately contained mistake.

The authors found that Fig. 4.B mistakenly displays an incorrect GAPDH image.

The authors are truly regretful and apologize for the mistake.

The online version of the original article can be found at https://doi.org/ $10.1007 / \mathrm{s} 12035-018-0868-4$

Antoni Camins

camins@ub.edu

1 Departament de Farmacología, Toxicologia i Quimica Terapéutica, Unitat de Farmacologia i Farmacognosia, Facultat de Farmacia i Ciències de l'Alimentació, Universitat de Barcelona, Av. Joan XXIII s/n, 08028 Barcelona, Spain

2 Biomedical Research Networking Center in Neurodegenerative Diseases (CIBERNED), Instituto de Salud Carlos III, Madrid, Spain

3 Unitat de Bioquímica i Biotecnologia, Facultat de Medicina I Ciències de la Salut, Universitat Rovira i Virgili, Reus, Tarragona, Spain

4 Institut de Neurociencias, Universitat de Barcelona, Barcelona, Spain

5 Unitat de Farmacia, Tecnologia Farmacèutica i Fisico-química, Facultat de Farmàcia i Ciències de l'Alimentació, Universitat de Barcelona, Barcelona, Spain

6 Institute of Nanoscience and Nanotechnology (IN2UB), University of Barcelona, Barcelona, Spain
Departamento de Biología Celular y Molecular, C.U.C.B.A, Universidad de Guadalajara and División de Neurociencias, Sierra Mojada 800, Col. Independencia, 44,340, Guadalajara, Jalisco, Mexico

8 Neurodegenerative Disorders Group, Instituto de Investigacion Hospital 12 de Octubre (i + 12), Madrid, Spain

9 Department of Biological Sciences, Kent State University, Kent, OH, USA

10 Departament de Biologia Cel·lular, Facultat de Biologia, Universitat de Barcelona, Barcelona, Spain

11 Institute of Biomedicine of the University of Barcelona (IBUB), Barcelona, Spain

12 Spanish Biomedical Research Center in Diabetes and Associated Metabolic Diseases (CIBERDEM)-Instituto de Salud Carlos III, Barcelona, Spain

13 Research Institute-Hospital Sant Joan de Déu, Esplugues de Llobregat, Barcelona, Spain 

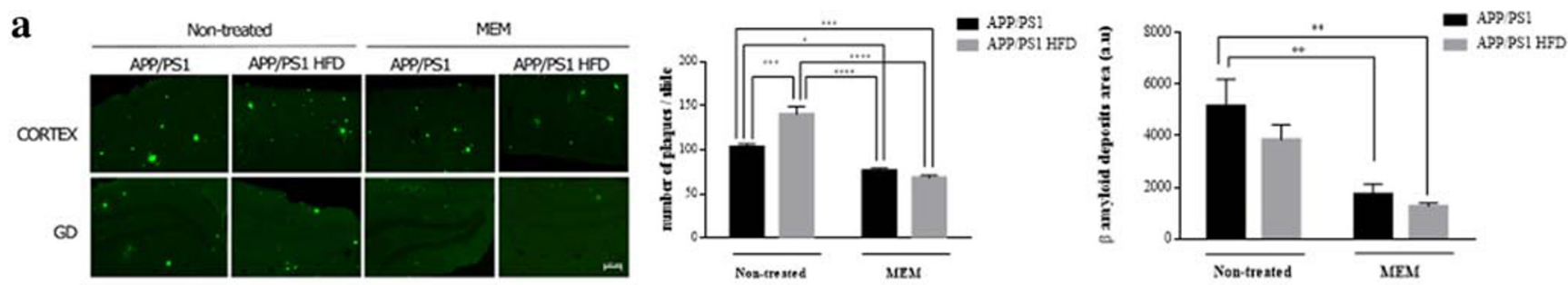

b
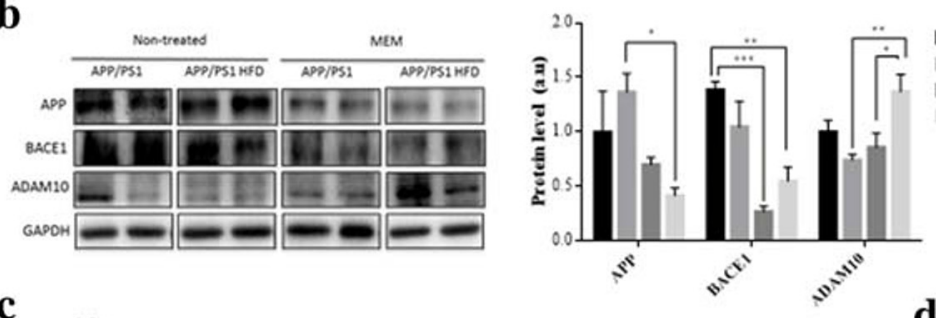

c
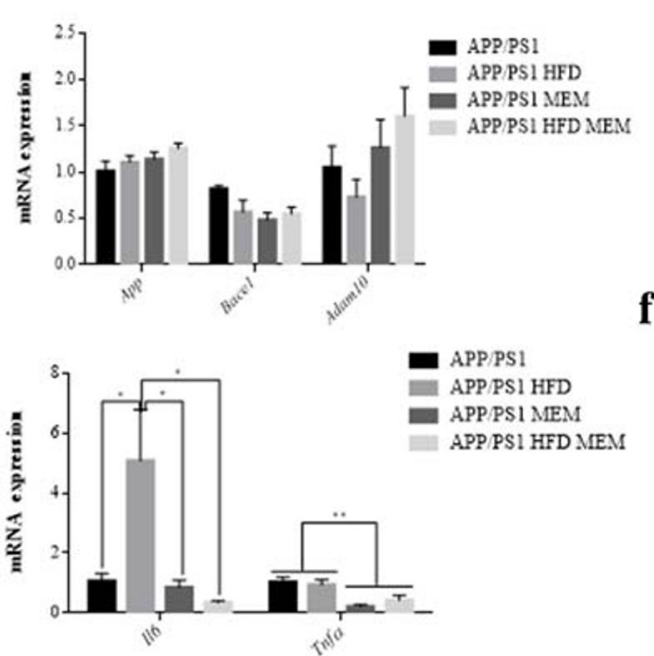

f

d
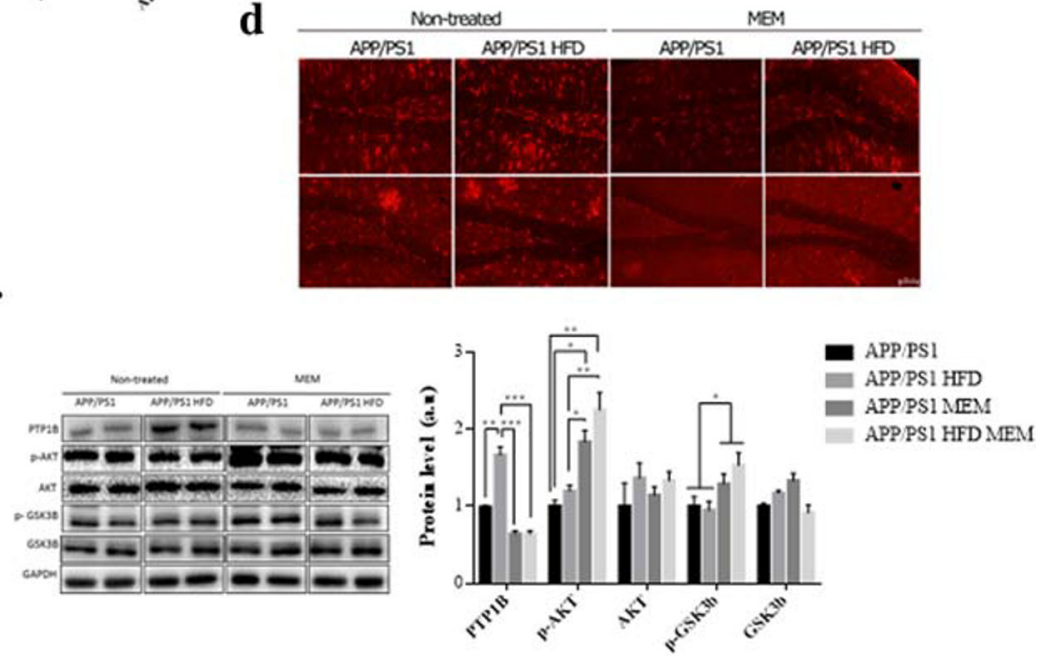